\title{
RESISTENCIA EMERGENTE A LOS ANTIBIÓTICOS: UNA AMENAZA GLOBAL Y UN PROBLEMA CRÍTICO EN EL CUIDADO DE LA SALUD
}

\author{
EMERGING ANTIBIOTIC RESISTANCE: A GLOBAL THREAT AND CRITICAL \\ HEALTHCARE PROBLEM \\ Claudio Rocha ${ }^{1, a}$, Nathanael D. Reynolds ${ }^{1, b}$, Mark P. Simons ${ }^{1, b}$ \\ U.S. Naval Medical Research Unit Nº, Callao, Perú \\ Médico cirujano ${ }^{\mathrm{b}} \mathrm{PhD}$ \\ Recibido: 11-02-15; Aprobado: 18-03-15
}

\begin{abstract}
RESUMEN
Después del desarrollo y la comercialización en masa de los antibióticos, las bacterias patógenas y ambientales han desarrollado resistencia a los antibióticos desde el siglo pasado, de modo que la infección causada por organismos resistentes a los antibióticos (ORAs) podría ser considerada como una infección emergente. Debido a ello, su control debe ser priorizado ya que constituye una amenaza para todas las naciones, sin reparar en su territorio y situación económica. El incremento de la vigilancia en Estados Unidos de América, Europa y Asia Oriental ha ilustrado lo rápido que pueden diseminarse, trayendo como consecuencia un incremento en la carga de infecciones causadas por los ORAs, sin embargo, la información disponible en los países de continuo desarrollo en América Latina es limitada. Esta revisión describe información reciente de estudios de vigilancia de ORAs en América Latina, así como también fuentes comunes de ORAs y posibles estrategias para su control.
\end{abstract}

Palabras clave: Resistencia bacteriana a antibióticos; Infecciones nosocomiales; Antibacterianos (fuente: DeCS BIREME).

\begin{abstract}
After the development and mass commercialization of antibiotics, pathogenic and environmental bacteria have developed resistance to antibiotics since the last century, so that the infection caused by antibiotic-resistant organisms (AROs) could be considered an emerging infection. As a result, its control should be prioritized as a threat to all nations, regardless of territory and economic situation. Increased surveillance in the United States, Europe and East Asia has illustrated the rapid spread leading to an increasing burden of infections caused by AROs. However, the information available in countries of continued development in Latin America is limited. This review describes recent information on AROs surveillance studies in Latin America as well as common sources of AROs and possible strategies for their control.
\end{abstract}

Key words: Antibiotic resistance, bacterial; Cross Infection; Anti-bacterial agents (source: MeSH NLM).

\section{INTRODUCCIÓN}

Las infecciones causadas por organismos resistentes a los antibióticos (ORAs) podrían llegar a ser consideradas como una infección emergente, debido a que su tratamiento es cada vez más limitado con el potencial de afectar a todas las personas en el mundo, tanto en países con mayores recursos económicos como en aquellos en vías de desarrollo. Al igual que cualquier enfermedad en un mundo globalizado, con modernas rutas de comercio y alto volumen de tráfico aéreo, las enfermedades emergentes en un lado del mundo se pueden diseminar prácticamente por todo el planeta en tiempo real. Los ORAs no son diferentes, constituyéndose como el "elefante blanco en la habitación", lo que significa que es un problema que crece en tamaño y tiene la habilidad de derribar todo el sistema de cuidado de la salud del mundo si no se aborda de forma colectiva por todas las personas y naciones. De acuerdo con los últimos cálculos del Centro para la Prevención y Control de Enfermedades de los Estados Unidos (CDC), los ORAs causan 2 millones de infecciones y 23000 muertes solo en los Estados Unidos cada año, con un impacto económico de $\$ 35$ millones adicionales de gastos en salud (1). Es probable que esta información este subreportada, pudiendo ser igual o mayor en otras regiones del mundo, en particular en países de continuo crecimiento tales como los de América Latina.

Citar como: Rocha C, Reynolds ND, Simons MP. Resistencia emergente a los antibióticos: una amenaza global y un problema crítico en el cuidado de la salud. Rev Peru Med Exp Salud Publica. 2015;32(1):139-45. 
El uso de antibióticos en la atención en salud es reciente en la historia de la humanidad con el descubrimiento de la penicilina en 1928, seguido por el uso extenso durante la Segunda Guerra Mundial. Poco después, el Staphylococcus desarrolló resistencia a la penicilina lo que inició el surgimiento de resistencia cuya tendencia se aceleró en las décadas subsiguientes.

En estos días es común encontrar aislamientos bacterianos tanto en el entorno clínico como en el ambiente con diferentes niveles de resistencia tales como los multidrogorresistentes (MDR; resistente a 2 o más antibióticos), extremadamente resistentes (XDR; resistente a 3 o más antibióticos), y aun más perturbador, aislamientos panresistentes, los cuales son literalmente intratables con los regímenes farmacológicos actuales, incluyendo terapias combinadas. La estrategia ha sido continuar la búsqueda de nuevos antibióticos naturales así como desarrollar modificaciones sintéticas de antibióticos existentes para recuperar eficacia. Sin embargo, al parecer la bacteria es "más inteligente" que nosotros, debido a que es capaz de crecer rápidamente, posee habilidades naturales de adquirir material genético foráneo como genes de resistencia, o porque presenta elevadas tasas de mutación inherentes durante la replicación genómica, de tal manera que han seguido adaptándose a las nuevas presiones selectivas desarrolladas principalmente por el uso indiscriminado de los antibióticos, por lo que su eficacia en el uso clínico se limita cada vez más.

Esta revisión resume el conocimiento actual que se tiene sobre los ORAs, detallando su aparición junto con la más actualizada información sobre su distribución geográfica en América Latina. También describe las fuentes en las que se originan estos patógenos y adquieren resistencia, y finalmente se discute brevemente la problemática de las estrategias y políticas ya implementadas en nuestra región e iniciativas de otros países orientadas a desarrollar medidas eficaces que podrían ser adoptadas por los gobiernos de América Latina para combatir este problema y así implementar políticas más eficaces enfocadas en las fuentes y epidemiología específica de los ORAs de nuestra región.

\section{DISTRIBUCIÓN GEOGRÁFICA DE LA EMERGENCIA DE BACTERIAS RESISTENTES A LOS ANTIBIOTICOS}

La información disponible acerca de la distribución geográfica y datos clínico-epidemiológicos de los ORAs proviene de las regiones ricas en recursos, siendo limitada en las regiones en desarrollo debido al subregistro en hospitales y en la comunidad, lo que ha conllevado a políticas regulatorias insuficientes para el control del uso de antibióticos en estos países.

La mayor parte de la información de los ORAs en América Latina proviene principalmente del análisis epidemiológico de infecciones nosocomiales, exhibiendo el surgimiento de un amplio espectro de resistencia a $\beta$-lactamasas durante el siglo XXI. Un reporte mostró la resistencia de E. coli en heces de niños saludables en Bolivia y Perú demostrando la presencia de genes de bla $a_{\text {СTX-M-2 }}$ y bla $a_{\text {СTX-M-15 }}$ responsables de la actividad de las $\beta$-lactamasas de expectro extendido (BLEE), proporcionando información de un indicio temprano de la emergencia de BLEE en estos países ${ }^{(2)}$. Igualmente, actividades realizadas por el programa de vigilancia de antibióticos SENTRY mostró que el $46 \%$ de las Klebsiella spp. causantes de infección del tracto urinario en pacientes hospitalizados en América Latina fueron BLEE, lo que demostró una alta prevalencia de resistencia en infecciones de alta frecuencia ${ }^{(3)}$. De forma similar, el análisis de los Enterobacteriaceae en hospitales de Brasil entre 1997 y 2003 demostraron que el $50 \%$ de las K. pneumoniae y el $18 \%$ de las E. coli eran positivas para BLEE ${ }^{(4)}$. Más recientemente, una revisión sistemática de la literatura publicada entre el 2005 y 2013 sobre aislamientos de Enterobacteriaceae de infecciones nosocomiales en América Latina demostraron que el $58 \%$ de las $K$. pneumoniae y el $32 \%$ de las E. coli eran productoras de BLEE, reportados colectivamente por un número de programas de vigilancia regional ${ }^{(5)}$. Recientemente, un estudio de vigilancia en coordinación entre 11 países de América Latina, incluyendo Perú, demostró altas tasas de prevalencia total de $E$. coli y de K. pneumoniae positivas para BLEE, Gram negativos resistentes a carbapenemasas, Enterococos resistentes a vancomicina (ERV), y Staphylococcus aureus resistente a meticilina (SARM) con hallazgos para Perú del 54\%, $70 \%, 0 \%, 16 \%$, y $79 \%$, respectivamente ${ }^{(6)}$. Cabe resaltar que en la mayoría de países considerados para este estudio participó solo un hospital (incluyendo Perú con 194 cepas), lo cual constituye una limitación a tomar en cuenta si queremos entender completamente la carga de ORAs en los países de nuestra región. Los SARM y las tasas de resistencia a BLEE entre los Enterobacteriaceae, se estan incrementando en América Latina y constituye una de las tasas de prevalencia más altas reportadas a nivel mundial, lo cual demuestra la importancia de entender completamente el surgimiento y diseminación de los factores de resistencia en nuestra región.

Las metalo- $\beta$-lactamasas (MBL) han surgido como la clase de carbapenemasas que se expande más rápidamente, y que comprende una amplia diversidad genética, cuya expresión se ha encontrado 
predominantemente en $P$. aeruginosa, Acinetobacter spp. y recientemente en Enterobacteriaceae. La alarmante velocidad de la diseminación de los tipos de MBL ha conllevado a una distribución global de las mismas, de tal manera que hoy en día los aislamientos positivos portadores de MBL estan siendo reportados en todos los continentes. Recientemente, la nueva New Delhi metalo- $\beta$-lactamasa (NDM) ha demostrado la facilidad con la que se diseminan los ORAs, descrita inicialmente en la India en 2008, para posteriormente expandirse rápidamente a través de toda Europa ${ }^{(7)}$. Se ha reportado resistencia a la carbapenemasa en Colombia, Perú, Chile, Brasil, Argentina, y Venezuela, así como también en Centro América ${ }^{(8-11)}$, pero a la fecha no han sido descritos aislamientos positivos para NDM en América del Sur. El programa de vigilancia de antibióticos SENTRY ha reportado la expresión de factores genéticos selectos de MBL incluyendo bla ${ }_{\text {IMP-1, }}$, bla $a_{\mathrm{IMP}-16}$, bla $a_{\mathrm{VIM}-2}$ y bla ${ }_{\mathrm{SPM}-1}$ en países de América Latina, encontrando positivos para MBL al $21 \%$ de aislamientos de Acinetobacter spp. y al $45 \%$ de aislamientos de $P$. aeruginosa ${ }^{12)}$. Así mismo, una revisión más reciente mostró que la resistencia a carbapenems en infecciones nosocomiales de América Latina expresaba genes de MBL en cepas de $P$. aeruginosa provenientes de México, Argentina, Brasil, Chile y Colombia (13). Por otro lado, un estudio de resistencia a imipenem por detección fenotípica de MBL en aislamientos bacterianos demostró que más del $70 \%$ de las $P$. aeruginosa provenientes de solo un establecimiento de salud en Sao Paulo, Brasil, era positiva a MBL ${ }^{(12)}$. Más aun, Argentina, Brasil y Colombia han reportado una predominancia del gen bla $_{\text {OxA }}$ clase D en los Acinetobacter spp. causantes de infecciones nosocomiales de estos países ${ }^{13}$ 14; sin embargo, solo dos reportes provenientes de Argentina y Brasil han identificado expresión $\mathrm{MBL} b \mathrm{bla}_{\mathrm{IMP}}$ en Acinetobacter spp. ${ }^{(15)}$. Aunque los reportes descritos todavía son escasos, demuestran la alta prevalencia de BLEE y MBL en los países de América Latina que han realizado estudios de los ORAs, por lo que es cada vez más necesaria la implementación de vigilancias sostenibles y eficaces en colaboración con todos los países de nuestra región.

\section{FUENTES DE BACTERIAS RESISTENTES A LOS ANTIBIÓTICOS Y PRESIONES EVOLUCIONARIAS SELECTIVAS MEDIOAMBIENTALES}

Se conoce que el entorno clínico constituye una fuente de resistencia a los antibióticos, debido al uso ampliamente extendido de los mismos, que produce una presión natural selectiva en las bacterias. La aparición de resistencia a los antibióticos en los países en desarrollo es una preocupación en todo el mundo, debido al uso no regulado de antibióticos en hospitales y lugares de suministro de medicamentos (farmacias, supermercados, y el mercado negro) de estos países, que va de la mano con la selección inadecuada de medicamentos, la dosificación equivocada, y la mala adherencia del paciente al tratamiento, lo que representa un escenario perfecto para el cultivo de bacterias resistentes ${ }^{(16)}$. Aunque existe la necesidad de seguir mejorando las vigilancias y desarrollando estudios en el entorno clínico, los ORAs han sido descritos principalmente en infecciones nosocomiales en el Perú y otros países de América Latina, encontrándose generalmente asociados a dispositivos médicos permanentes como catéteres. Existe limitada información acerca de las fuentes de ORAs causantes de infección provenientes de la comunidad ${ }^{(17,18,19)}$.

Recientemente, se ha incrementado la atención hacia las fuentes ambientales de presión selectiva de antibióticos. El tratamiento de las aguas residuales de hospitales, municipios, la industria farmacéutica y la agricultura también han sido reconocidos como fuentes de exposición ambiental a los antibióticos y a genes de resistencia antibiótica (GRAs) ${ }^{(20)}$. Varios estudios han destacado que la contaminación por antibióticos en el medioambiente, acumulados durante más de 60 años de uso de antibióticos por los seres humanos desde el descubrimiento de la penicilina, ha promovido la evolución independiente de elementos de resistencia específicos en bacterias patógenas y no patógenas, o bacterias oportunistas (21). Es bien reconocido que la transferencia horizontal de genes (THG) entre diferentes especies de bacterias es el mecanismo mediante el cual las bacterias adquieren genes de resistencia a los antibióticos, por lo que varios autores han sugerido que los determinantes de la resistencia podrían originarse y diseminarse en el ambiente mediante estos mecanismos (20). Aunque se conoce poco respecto al mecanismo y origen de los GRAs en el ambiente, el rápido ritmo de aumento de la resistencia a los antibióticos en el entorno clínico sugiere una reserva preexistente de GRAs en los reservorios naturales medioambientales del mundo ${ }^{(22)}$. Un estudio realizado en Canadá mostró que una variedad de bacterias resistentes no patogénicas aisladas de zonas urbana, agrícola, y forestal fueron capaces de inactivar daptomicina $(80 \%)$, rifampicina $(40 \%)$, synercid $(18 \%)$ y ciprofloxacina $(11 \%)$, lo que sugiere desarrollo de resistencia en ausencia de presiones selectivas ambientales. En este punto del estudio, la daptomicina había sido recién aprobada por la FDA y su uso no había sido masificado, lo que sugiere que la resistencia, sea 
inherente o adquirida ambientalmente, puede existir en la ausencia de fuentes humanas (21). Estudios en regiones remotas, tales como la selva amazónica, han demostrado infecciones resistentes a los antibióticos en personas que no han estado expuestas a la civilización moderna y los medicamentos ${ }^{(23)}$. Se necesitaría una vigilancia adicional en zonas urbanas y entornos remotos para clarificar la contribución humana versus verdaderas fuentes ambientales de resistencia a los antibióticos.

Es bien conocida la contribución de la agricultura, el ganado y en general los animales destinados a consumo en la contaminación del medioambiente con antibióticos, y se ha convertido en una preocupación creciente en todo el mundo, debido al hecho de que tales antibióticos pertenecen a la misma clase de los utilizados en los seres humanos (como tetraciclinas y aminoglicósidos) ya que son administrados en grandes cantidades a dichos animales para promover su crecimiento ${ }^{(24)}$. Por ejemplo, solo en los Estados Unidos (EE. UU.), Australia y China se ha estimado que la producción total de antibióticos administrados al ganado es igual o mayor a lo administrado en humanos (20), así mismo, se estima que el $80 \%$ de los 8 millones de pollos destinados a consumo humano cada año en Ios EE. UU. son tratados con gentamicina para prevenir muerte temprana ${ }^{(25)}$. Cada vez hay más evidencia de que el uso de antibióticos en animales destinados al consumo genera un depósito de ORAs y GRAs que se pueden propagar a la población humana, y que, a su vez, limita el valor médico de dichos antibióticos. En respuesta a este problema, la Unión Europea (UE) ha prohibido el uso de antibióticos como aditivos en forrajes para estimular el crecimiento de estos animales, debido a la evidencia de resistencia cruzada a los agentes antimicrobianos utilizados en tratamientos en humanos (24). Estas regulaciones han mitigado en gran medida los ORAs y los GRAs provenientes de la utilización de animales en la UE, lo que demuestra que es posible revertir la aparición de resistencia a los antibióticos en la población de animales destinados al consumo ${ }^{(20)}$.

El uso de antibióticos en el ganado conduce a una gran cantidad de antibióticos en estiércol y consecuentemente a tasas altas de GRAs y ORAs diseminadas en agua y suelo. Se pueden adoptar y desarrollar varias medidas para prevenir infecciones en granjas y la diseminación de ORAs en el ambiente, tales como rebajar la densidad de animales en las mismas, programas nutricionales mejorados en animales para reducir la necesidad de tratamiento con antibióticos durante el crecimiento, así como el manejo adecuado del estiércol usando métodos como la fermentación, compostaje y contención de residuos animales, prevención de derrames y filtraciones, control del drenaje en la superficie de los suelos, limitar la erosión de sedimentos, y el tratamiento anaeróbico ${ }^{(20)}$.

Otra fuente potencial de antibióticos contaminantes y ORAs en el medio ambiente es la acuicultura, que similar a la industria ganadera, utiliza antibióticos para promover el stock de peces saludables ${ }^{(20)}$. Los países desarrollados han impuesto estrictas regulaciones del uso de antibióticos en la acuicultura, sin embargo, la mayoría de la producción global acuícola se produce en países en vías de desarrollo donde no hay regulaciones bien diseñadas del uso de antibióticos en este campo. Hay pruebas de que algunas bacterias presentes en el agua y en el pescado, especialmente cuando se exponen a las aguas residuales de humanos y animales, desarrollan mecanismos de resistencia a los antibióticos ${ }^{(20,26)}$. La acuicultura podría representar una de la principales vías para difundir y transportar los ORAs, debido al hecho que los países desarrollados importan grandes cantidades de pescado y mariscos provenientes del extranjero; por ejemplo la UE importa aproximadamente $40,8 \%$ de los mariscos del mundo y Ios EE. UU. y Japón juntos el 27,2\% del total mundial ${ }^{(20)}$.

Por último, el saneamiento básico tanto de residuos humanos como de animales es una preocupación en todo el mundo, no solo para mejorar la higiene y reducir las enfermedades diarreicas, sino también por el potencial de la rápida propagación de los ORAs a través de la liberación en el medioambiente y el agua del suelo. Por ejemplo, gran cantidad de antibióticos se liberan en las plantas de tratamiento de aguas residuales como resultado de utilizar antibióticos en los hogares, instalaciones de producción farmacéuticas y hospitales, con un enorme potencial para ejercer una presión selectiva sobre las bacterias del medioambiente para adquirir GRAs. Los antibióticos que están mal metabolizados y no completamente absorbidos por el tracto digestivo como las tetraciclinas, son de particular interés ya que las presiones ambientales, tales como la acumulación de aguas de desagüe en plantas de tratamiento de aguas residuales (PTARs), se convierten en grandes reservorios de antibióticos debido a que la mayoría de las plantas de tratamiento tradicionales no están diseñadas para la eliminación de antibióticos y GRAs ${ }^{(20,27)}$. Los PTARs parecen ser el ambiente ideal para mejorar la transferencia horizontal de genes y el desarrollo de los ORAs ${ }^{(2028,29)}$. Estudios recientes han demostrado efectos negativos como resultado de una exposición sutil pero de largo plazo a los productos farmacéuticos en los microorganismos acuáticos y terrestres, incluyendo los efectos de la exposición a los 
antibióticos que están contaminando el medioambiente, y que suministran presión selectiva para la adquisición de GRAs ${ }^{(27)}$. Por lo tanto, un enfoque combinado de los programas de administración de antibióticos en el ambiente clínico y de agricultura, así como el desarrollo de ingeniería para mejorar el saneamiento y tratamiento de aguas residuales (por ejemplo control de antibióticos y GRAs) serían necesarios para reducir las presiones selectivas del medio ambiente en el desarrollo de resistencia a los antibióticos.

\section{PROGRESOS ACTUALES: ACCIONES PARA COMBATIR EL PROBLEMA DE LAS INFECCIONES RESISTENTES A LOS ANTIBIÓTICOS}

Los esfuerzos para la implementación de vigilancia y control de las ORAs por parte de organismos internacionales tales como la Organización Mundial de la Salud (OMS), el Centro de Control de Infecciones de los Estados Unidos (CDC), el programa SENTRY, el Consorcio Internacional de Control de Infecciones Nosocomiales (INICC por sus siglas en inglés), y muchas otras, han sido insuficientes para conocer la verdadera carga de los ORAs y mitigar el problema de forma global. Un ejemplo de ello, es la falta de muestras representativas en los países estudiados ${ }^{(6)}$. Esto solo permite un conocimiento parcial de la verdadera carga de infecciones producidas por los ORAs. El problema surge probablemente, como consecuencia de la falta de mecanismos adecuados por parte de los gobiernos para establecer colaboraciones efectivas para la vigilancia y control de los ORAs entre los países de la región, que permita una estrategia con un enfoque global. Otro problema ha sido la inadecuada implementación de las guías de vigilancia y control propuestas por dichos organismos internacionales. No han tenido éxito debido a la falta de políticas efectivas en cada país que viabilicen económica, logística y administrativamente dichas implementaciones. La solución no solo recae en los hacedores de políticas de los países de la región, para comprometerse a viabilizar estas acciones mediante políticas claras, oportunas y estrictas, sino también por parte del personal de salud de todos los niveles para cumplir los protocolos y advertir a las autoridades del problema creciente de forma oportuna.

En una acción sin precedentes de voluntad política frente a este problema, el presidente de los Estados Unidos, Barack Obama emitió una Orden Ejecutiva en septiembre de 2014 para combatir las infecciones producidas por los ORAs, dedicando fondos especiales y destacando los elementos de acción mencionados en la Estrategia Nacional, así como el nombramiento de un grupo de trabajo especial de expertos para supervisar la ejecución de estas iniciativas para el año 2020 (30). Los puntos que se destacan en la Estrategia Nacional de Estados Unidos para combatir las infecciones resistentes a los antibióticos incluyen: 1) contener la emergencia y prevenir la propagación de bacterias resistentes, 2) fortalecer los esfuerzos nacionales para la identificación y reporte de casos de resistencia antibiótica, 3) avanzar con el desarrollo y uso de pruebas diagnósticas para la identificación y caracterización de bacterias resistentes a los antibióticos, 4) acelerar la investigación básica y aplicada para desarrollar nuevos antibióticos, otras terapias y vacunas, 5) mejorar la colaboración internacional y las capacidades para la prevención de resistencia a los antibióticos, así como también mejorar la vigilancia, control e investigación y desarrollo de nuevos antibióticos. El último objetivo es especialmente importante, porque reconoce a los ORAs como problema mundial y para contener su emergencia se debe alcanzar una colaboración que permita suficiencia e igualdad de capacidades de manera global.

La prioridad es asegurar el establecimiento de adecuados mecanismos de vigilancia y de forma escalonada permitir el desarrollo tanto de una vigilancia centinela como de un reporte oportuno para tomar acciones apropiadas. Se necesita una vigilancia de los ORAs que sea geográficamente amplia (que puede estar dividida en nacional, continental o mundial), sostenible en el tiempo y estandarizada en sus procesos epidemiológicos y laboratoriales, especialmente en regiones remotas y en aquellas donde existe más riesgo que la presión selectiva cause el surgimiento de nueva resistencia. Para ello, se requerirá incluir una adecuada infraestructura laboratorial, personal entrenado y competente, así como la habilidad de integrar información y tecnología nueva. Así mismo, su implementación requerirá el compromiso de las autoridades de cada país, principalmente de aquellos que se encuentran en un continuo desarrollo económico y en los que la distribución de los recursos es todavía inequitativa, especialmente lo destinado a salud y educación.

La mayor limitación, no solo en países desarrollados sino especialmente en países de bajos recursos, es el financiamiento para apoyar las capacidades laboratoriales y programas de entrenamiento que provean carreras adecuadas a los profesionales en laboratorio que les permita ser capaces de apoyar a estas iniciativas de forma local. Es necesario que haya suficiente personal establecido en cada región para evitar abrumar a este personal con sobrecarga de trabajo, lo que muy frecuentemente conlleva a errores 
en los resultados y reportes. Nunca antes hemos tenido mayor presión por la necesidad de profesionales en laboratorio, estadísticos y epidemiólogos, por lo tanto, es necesario que los gobiernos apoyen programas universitarios de entrenamiento para estas profesiones en todos los niveles, desde técnicos en laboratorio hasta estadísticos y científicos. Además del entrenamiento y apoyo al personal, es importante integrar nuevas tecnologías que permitan datos con reportes más precisos y oportunos provenientes de la vigilancia, manteniéndose al día con la rápida evolución de la resistencia antibiótica y que, a su vez, requiere de la combinación de las antiguas pruebas fenotípicas y de las más avanzadas técnicas moleculares para detectar nuevas variantes genotípicas. También se debe priorizar los mecanismos para el aseguramiento de la calidad y del control de calidad para las pruebas de laboratorio y el procesamiento de datos, asegurando la precisión de la información obtenida.

Además de la vigilancia, las naciones, ciudades y personas, deben estar dispuestas a modificar comportamientos y prácticas para adaptarse efectivamente a este creciente problema de la resistencia antibiótica, incluyendo cambios en el comportamiento de la búsqueda de atención, menos visitas de atención para enfermedades benignas que no requieren terapia antibiótica, y evitar la autoadministración de antibióticos en situaciones en que la evidencia ha demostrado un impacto pequeño (ej. enfermedad respiratoria aguda que es muy comúnmente de origen viral), pero también se debe buscar atención para situaciones severas que requieren terapia agresiva (ej. infecciones del torrente sanguíneo). Los profesionales de la salud, en general, necesitan educar a sus pacientes, proporcionando información acerca de cuándo es el momento adecuado para buscar atención y las formas de hacerlo (visitas para atención primaria vs departamentos de emergencia). Se debe globalizar la educación para que sea efectiva, de tal forma que todos (tanto el personal de salud como los ciudadanos) puedan participar en los esfuerzos globales contra los ORAs.

Finalmente, la evidencia sugiere cada vez más, que existen fuentes ambientales de ORAs, por lo que las estrategias deberían incluir también un mejor diseño de sistemas de tratamiento de aguas residuales en hospitales y municipalidades, así como también el desarrollo de políticas estrictas no solo para adecuados programas de administración de antibióticos en el entorno clínico, sino también para el uso de antibióticos en la cría de animales y en la disposición de sus residuos.

Pasar por alto el problema creciente de la resistencia antibiótica, conllevará a pobres resultados en la atención en salud y pérdidas de las capacidades económicas, debido al aumento en los costos en la atención y pérdidas en días de trabajo y productividad de personas enfermas. Una planificación efectiva y oportuna puede salvar millones de vidas y miles de millones de dólares, por lo que se convierte en un paso clave para desarrollar medidas sostenibles y contener esta amenaza global ahora y en el futuro.

\section{Contribuciones de autoría:}

Fuentes de financiamiento: autofinanciado.

Conflictos de interés: los autores declaran no tener conflictos de interés.

\section{REFERENCIAS BIBLIOGRÁFICAS}

1. Centers for Disease Control and Prevention. Antibiotic Resistance Threats in the United States. 2013. Chicago: CDC; 2014.

2. Pallecchi L, Malossi M, Mantella A, Gotuzzo E, Trigoso C, Bartoloni A, et al. Detection of CTX-M-type betalactamase genes in fecal Escherichia coli isolates from healthy children in Bolivia and Peru. Antimicrob Agents Chemother. 2004 Dec;48(12):455661.

3. Gales AC, Sader HS, Jones RN; SENTRY Participants Group (Latin America). Urinary tract infection trends in Latin American hospitals: report from the SENTRY antimicrobial surveillance program (1997-2000). Diagn Microbiol Infect Dis. 2002 Nov;44(3):289-99.

4. Sader HS, Gales AC, Pfaller MA, Mendes RE, Zoccoli C, Barth A, et al. Pathogen frequency and resistance patterns in Brazilian hospitals: summary of results from three years of the SENTRY Antimicrobial Surveillance Program. Braz J Infect Dis. 2001 Aug;5(4):200-14.

5. Guzmán-Blanco M, Labarca JA, Villegas MV, Gotuzzo E; Latin America Working Group on Bacterial Resistance. Extended spectrum $\beta$-lactamase producers among nosocomial Enterobacteriaceae in Latin America. Braz J Infect Dis. 2014 Jul-Aug;18(4):421-33. doi: 10.1016/j. bjid.2013.10.005.

6. Jones RN, Guzman-Blanco M, Gales AC, Gallegos B, Castro AL, Martino MD, et al. Susceptibility rates in Latin American nations: report from a regional resistance surveillance program (2011). Braz J Infect Dis. 2013 Nov-Dec;17(6):672-81. doi: 10.1016/j.bjid.2013.07.002.

7. Johnson AP, Woodford N. Global spread of antibiotic resistance: the example of New Delhi metallo- 
$\beta$-lactamase (NDM)-mediated carbapenem resistance. J Med Microbiol. 2013 Apr;62(Pt 4):499513. doi: 10.1099/jmm.0.052555-0.

8. Gales AC, Castanheira M, Jones RN, Sader HS. Antimicrobial resistance among Gram-negative bacilli isolated from Latin America: results from SENTRY Antimicrobial Surveillance Program (Latin America, 2008-2010). Diagn Microbiol Infect Dis. 2012 Aug;73(4):354-60. doi: 10.1016/j. diagmicrobio.2012.04.007.

9. Reinert RR, Low DE, Rossi F, Zhang X, Wattal C, Dowzicky MJ. Antimicrobial susceptibility among organisms from the Asia/Pacific Rim, Europe and Latin and North America collected as part of TEST and the in vitro activity of tigecycline. J Antimicrob Chemother. 2007 Nov;60(5):1018-29.

10. Rossi F, Garcia P, Ronzon B, Curcio D, Dowzicky MJ. Rates of antimicrobial resistance in Latin America (20042007) and in vitro activity of the glycylcycline tigecycline and of other antibiotics. Braz J Infect Dis. 2008 Oct;12(5):405-15.

11. Fernandez-Canigia L, Dowzicky MJ. Susceptibility of important Gramnegative pathogens to tigecycline and other antibiotics in Latin America between 2004 and 2010. Ann Clin Microbiol Antimicrob. 2012 Oct 22;11:29. doi: 10.1186/1476-071111-29.

12. Sader HS, Castanheira M, Mendes RE, Toleman M, Walsh TR, Jones RN. Dissemination and diversity of metallo-beta-lactamases in Latin America: report from the SENTRY Antimicrobial Surveillance Program. Int J Antimicrob Agents. 2005 Jan;25(1):57-61.

13. Labarca JA, Salles MJ, Seas C, Guzman-Blanco M. Carbapenem resistance in Pseudomonas aeruginosa and Acinetobacter baumannii in the nosocomial setting in Latin America. Crit Rev Microbiol. 2014 Aug 27:1-17.

14. Opazo A, Dominguez M, Bello $H$, Amyes SG, Gonzalez-Rocha G. OXAtype carbapenemases in Acinetobacter baumannii in South America. J Infect Dev Ctries. 2012 Apr 13;6(4):311-6.

15. Fritsche TR, Sader HS, Toleman MA, Walsh TR, Jones RN. Emerging metallo-beta-lactamase-mediated resistances: a summary report from the worldwide SENTRY antimicrobial surveillance program. Clin Infect Dis. 2005 Aug 15;41 Suppl 4:S276-8.

16. Salles MJ, Zurita J, Mejía C, Villegas MV; Latin America Working Group on Bacterial Resistance. Resistant gramnegative infections in the outpatient setting in Latin America. Epidemiol Infect. 2013 Dec;141(12):2459-72. doi: 10.1017/S095026881300191X.

17. García C, Llamocca LP, García K, Jiménez A, Samalvides F, Gotuzzo E, et al. Knowledge, attitudes and practice survey about antimicrobial resistance and prescribing among physicians in a hospital setting in Lima, Peru. BMC Clin Pharmacol. 2011 Nov 15;11:18. doi: 10.1186/1472-6904-11-18.

18. Cuellar LE, Fernandez-Maldonado E, Rosenthal VD, Castaneda-Sabogal A, Rosales R, Mayorga-Espichan MJ, et al. Device-associated infection rates and mortality in intensive care units of Peruvian hospitals: findings of the International Nosocomial Infection Control Consortium. Rev Panam Salud Publica. 2008 Jul;24(1):16-24.

19. Salomao R, Rosenthal VD, Grimberg G, Nouer S, Blecher S, BuchnerFerreira $S$, et al. Device-associated infection rates in intensive care units of Brazilian hospitals: findings of the International Nosocomial Infection Control Consortium. Rev Panam Salud Publica. 2008 Sep;24(3):195202.

20. Pruden A, Larsson DGJ, Amézquita A, Collignon P, Brandt K, Graham $\mathrm{D}$, et al. Management Options for Reducing the Release of Antibiotics and Antibiotic Resistance Genes to the Environment. Environ Health Perspect. 2013;121(8):878-84.

21. D'Costa VM, McGrann KM, Hughes DW, Wright GD. Sampling the antibiotic resistome. Science. 2006 Jan 20;311(5759):374-7.

22. Nesme J, Simonet P. The soil resistome: a critical review on antibiotic resistance origins, ecology and dissemination potential in telluric bacteria. Environ Microbiol. 2014 Oct 6. doi: 10.1111/1462-2920.12631.

23. Bartoloni A, Pallecchi L, Rodríguez $\mathrm{H}$, Fernandez C, Mantella A, Bartalesi
$\mathrm{F}$, et al. Antibiotic resistance in a very remote Amazonas community. Int J Antimicrob Agents. 2009 Feb;33(2):125-9. doi: 10.1016/j. ijantimicag.2008.07.029.

24. Heuer OE, Hammerum AM, Collignon P, Wegener HC. Human health hazard from antimicrobialresistant enterococci in animals and food. Clin Infect Dis. 2006 Oct 1;43(7):911-6.

25. Donabedian SM, Thal LA, Hershberger E, Perri MB, Chow JW, Bartlett P, et al. Molecular characterization of gentamicin-resistant Enterococci in the United States: evidence of spread from animals to humans through food. J Clin Microbiol. 2003 Mar;41(3):110913.

26. Cabello FC. Heavy use of prophylactic antibiotics in aquaculture: a growing problem for human and animal health and for the environment. Environ Microbiol. 2006 Jul;8(7):1137-44.

27. Boxall ABA. The environmental side effects of medication. EMBO Rep. 2004 Dec;5(12):1110-6.

28. Kristiansson E, Fick J, Janzon A, Grabic R, Rutgersson C, Weijdegård $\mathrm{B}$, et al. Pyrosequencing of antibioticcontaminated river sediments reveals high levels of resistance and gene transfer elements. PLoS One. $2011 \mathrm{Feb}$ 16;6(2):e17038. doi: 10.1371/journal. pone.0017038.

29. LaPara TM, Burch TR, McNamara PJ Tan DT, Yan M, Eichmiller JJ. Tertiarytreated municipal wastewater is a significant point source of antibiotic resistance genes into Duluth-Superior Harbor. Environ Sci Technol. 2011 Nov 15;45(22):9543-9. doi: 10.1021/ es202775r.

30. Centers for Diseases Control and Prevention. National Strategy for Combatting Antibiotic-Resistant Bacteria. Atlanta: CDC; 2013.

Correspondencia: Claudio Rocha

Dirección: U.S. Naval Medical Research Unit $N^{\circ}$ 6, Bellavista $S / N$, Avenida Venezuela, Callao, Perú

Teléfono: (+51) 6144141

Correo electrónico: claudio.rocha@med.navy.mil 